Corrigendum

\title{
Corrigendum to "Pulsed Radiofrequency of the Trigeminal Ganglion for Treating Postherpetic Neuralgia of the Ophthalmic Branch"
}

\author{
Dong-Yang Liu, Jin-Sheng Chen, Ze-Zang Fang, Shao-Yan Liu, and Li Wan \\ Department of Pain Management, The State Key Clinical Specialty for Pain Medicine, The Second Affiliated Hospital, \\ Guangzhou Medical University, Guangzhou, Guangdong 510260, China
}

Correspondence should be addressed to Li Wan; wanli5000cn@163.com

Received 11 November 2021; Accepted 11 November 2021; Published 14 December 2021

Copyright (C) 2021 Dong-Yang Liu et al. This is an open access article distributed under the Creative Commons Attribution License, which permits unrestricted use, distribution, and reproduction in any medium, provided the original work is properly cited.

In the article titled "Pulsed Radiofrequency of the Trigeminal Ganglion for Treating Postherpetic Neuralgia of the Ophthalmic Branch" [1], the acknowledgement section should be corrected as follows:

"The authors thank Chong-Rong Gao and Zhen-He Lu for their initial help with the project and members of the Department of Pain Management for their comments. Moreover, special thanks are due to $\mathrm{Li} \mathrm{Hu}$ for the advice on this manuscript. The research was supported by the National Natural Science Foundation of China (No. 81771182) to Li Wan and by the High-Level University Construction Promotion Project of Guangzhou Medical University (2017-No. 160-9) to Li Wan."

\section{References}

[1] D. Y. Liu, J. S. Chen, Z. Z. Fang, S. Y. Liu, and L. Wan, “Pulsed radiofrequency of the trigeminal ganglion for treating postherpetic neuralgia of the ophthalmic branch," Pain Research \& Management, vol. 2021, Article ID 6638392, 6 pages, 2021. 